Article

\title{
Dynamics on Binary Relations over Topological Spaces
}

\author{
Chung-Chuan Chen ${ }^{1}$, J. Alberto Conejero ${ }^{2, *} \mathbb{D}$, Marko Kostić ${ }^{3}$ and Marina Murillo-Arcila ${ }^{4}$ \\ 1 Department of Mathematics Education, National Taichung University of Education, Taichung 403, Taiwan; \\ chungchuan@mail.ntcu.edu.tw \\ 2 Instituto Universitario de Matemática Pura y Aplicada, Universitat Politècnica de València, \\ E-46022 València, Spain \\ 3 Faculty of Technical Sciences, University of Novi Sad, Trg D. Obradovića 6, 21125 Novi Sad, Serbia; \\ marco.s@verat.net \\ 4 Departamento de Matemáticas, Universitat Jaume I, Campus de Ríu Sec, E-12071 Castelló de la Plana, Spain; \\ murillom@uji.es \\ * Correspondence: aconejero@upv.es
}

Received: 16 April 2018; Accepted: 7 June 2018; Published: 11 June 2018

check for updates

\begin{abstract}
The existence of chaos and the quest of dense orbits have been recently considered for dynamical systems given by multivalued linear operators. We consider the notions of topological transitivity, topologically mixing property, hypercyclicity, periodic points, and Devaney chaos in the general case of binary relations on topological spaces, and we analyze how they can be particularized when they are represented with graphs and digraphs. The relations of these notions with different types of connectivity and with the existence of Hamiltonian paths are also exposed. Special attention is given to the study of dynamics over tournaments. Finally, we also show how disjointness can be introduced in this setting.
\end{abstract}

Keywords: Devaney chaos; hypercyclicity; topological transitivity; topologically mixing; disjointness; connectivity

\section{Introduction}

One of the pillars of the study of chaos in dynamical systems is the search of orbits that are dense in the whole space. Typical examples of chaotic maps on the interval, where chaos can be easy visualized, are given by the tent map or by some functions of the logistic family (see, for instance, [1-4]).

The existence of orbits that spread along the whole space has been also studied in the setting of binary relations. When they are considered from one set onto itself, one can consider them from the point of view of graph theory. On the one hand, Hamilton was the first one who started to consider the analysis of graphs containing paths that visit all the nodes, named Hamiltonian paths. On the other hand, in terms of connectivity of (directed) graphs, a graph is (strongly) connected if, for every (ordered) pair of nodes, there is a path connecting them.

Roughly speaking, these two areas share the idea of the quest of orbits/paths visiting (nearly) the whole domain. But there are differences in how these problems are addressed. In the case of chaotic maps on the interval, the results usually involve computable conditions on the parameters of the function that defines the system. For instance, this is the case of how it is determined the chaos of the logistic map for $\mu=4$. However, in the case of graphs, these results are related with the global structure of edges/arcs of the graph and/or on the quantification of the local structure at every node.

In the present work, our goal is to investigate the dynamics on graphs and on the more general frame of binary relations on topological spaces. In this setting, when a relation is composed with another one (or itself), each element of the domain does not need to be necessarily connected with a single element in the range. For this reason, we have set a connection with some recent results of the 
authors that were introduced to study the dynamics of multivalued continuous linear operators [5]. We will analyze hypercyclicity, topological transitivity, and topologically mixing properties of binary relations. It is worth mentioning that the study of dynamics over finite graphs has been recently considered by Bahi et al. by setting links between Devaney chaos and strong connectivity in order to provide an algorithm for the generation of strongly connected graphs and to construct Pseudo Random Number Generators (PRNGs) [6]. This approach has also allowed for the obtainment of PRNGs based on the construction of Hamiltonian cycles over an $\mathrm{N}$-cube [7,8]. It is also worth mentioning that such results can also be considered in connection with finite state machines [9] and explained using Turing machines [10].

In the present work, we have also tackled the problem of the link between chaotic properties and connectivity, but analyzing more carefully the implications of using different topologies over the set of nodes. These results will allow us to stretch the connections between graph theory and dynamical systems in order to facilitate the exchange of ideas between both areas.

It is worth mentioning that there have been also recent results concerning the dynamics of continuous linear operators acting on $L^{p}$-spaces consisting of functions $V \mapsto \mathbb{K}$, where $\mathbb{K}=\{\mathbb{R}, \mathbb{C}\}$, and $V$ is a multigraph, an infinite or a Cayley graph, a tree, or some similar structure (see, e.g., [11,12] and the references cited therein). We point out that our approach is different to the one of studying associations of directed graphs with finite topologies, as is taken in [13]. It also differs from the one taken by Namayanja; she generalized the dynamics of solution $C_{0}$-semigroups of birth-and-death models [14-16] to the case in which the transport equations are defined on the edges of an infinite network [17].

\section{Preliminaries}

Given two nonempty sets $X$ and $Y$, a binary relation $E$ is a subset of the Cartesian product $X \times Y$. If we consider the following relations $\rho \subseteq X \times Y$ and $\sigma \subseteq Z \times T$ with $Y \cap Z \neq \varnothing$, then we can define the inverse of $\rho$, denoted by $\rho^{-1} \subseteq Y \times X$, as the relation $\rho^{-1}:=\{(y, x) \in Y \times X:(x, y) \in \rho\}$, and the composition of relations $\sigma \circ \rho \subseteq X \times T$ by

$$
\sigma \circ \rho:=\{(x, t) \in X \times T: \exists y \in Y \cap Z \text { such that }(x, y) \in \rho \text { and }(y, t) \in \sigma\} .
$$

Given $x \in X$, we define its set of adjacent elements by $\rho(x):=\{y \in Y:(x, y) \in \rho\}$. The domain of a binary relation $\rho \subseteq X \times Y$ is given by $D(\rho):=\{x \in X: \exists y \in Y$ such that $(x, y) \in \rho\}$ and the range of $\rho$ is defined as $R(\rho):=\{y \in Y: \exists x \in X$ such that $(x, y) \in \rho\}$.

We define the $n$-th power of $\rho$ as $\rho^{n}:=\underbrace{\rho \circ \cdots \circ \rho}_{n}$, their inverses $\rho^{-n}:=\left(\rho^{n}\right)^{-1}$, and the trivial relation $\rho^{0}:=\Delta_{X}:=\{(x, x): x \in X\}$. We also set $D_{\infty}(\rho):=\bigcap_{n \in \mathbb{N}} D\left(\rho^{n}\right)$ and $\mathbb{N}_{n}:=\{1, \ldots, n\}$, for every $n \in \mathbb{N}$. The definitions of the reflexive, symmetric, anti-symmetric, and transitive properties are assumed to be known, as long as the classes of equivalence and partial order relations.

When $X=Y$, a binary relation can be also understood as the links of a graph. If we distinguish the order in which the elements appear in each pair of the relation, then we speak of a directed graph or a digraph; if not, we will refer to it as an undirected graph or simply as a graph. Following the previous notation, a (di-)graph $G=(X, \rho)$ is given by a nonempty set $X$, whose elements are called nodes or vertices, and a set $\rho$ or (ordered) pairs of elements of $X$, called arcs in the directed case and edges in the undirected one. Thus, a binary relation on a graph is just the set of arcs/edges.

If $y \notin \rho(x)$, then $y$ is not adjacent to $x$. In a graph, we define the degree of $x$ as the cardinal of $\rho(x),|\rho(x)|$. In a digraph, the outer degree of $x, d^{+}(x)$, is given by $|\rho(x)|$, and the inner degree of $x$, $d^{-}(x)$, is given by $|\{y \in Y:(y, x) \in \rho\}|$. The set $X$ of nodes of a (di-)graph $G$ can be considered as a topological space when endowed with certain topology over its elements. In the sequel, we will only deal with non-trivial finite simple (di-)graphs (without multiple edges connecting two nodes, and without any pair $(x, x)$ in $\rho)$. 
A walk is an ordered sequence of nodes $x_{1}, \ldots, x_{n+1} \in X$ such that $\left(x_{i}, x_{i+1}\right) \in \rho, 1 \leq i \leq n$. In this case, we say that its length is $n$. A path is a walk that does not include any node twice, except that its first node can be the same as the last one. Such a (walk) path is called a $x_{1}-x_{n+1}$ (walk) path. A path with $x_{1}=x_{n+1}$ is called a closed path or a cycle. Given an element $x \in X$, we define its set of accessible elements by $\omega(x):=\{y \in Y:$ there is an $x-y$ path $\}$. We say that a (di-)graph is (strongly) connected if, forevery pair of nodes $x, y \in X$, there is an $x-y$ path. A digraph whose underlying non-directed graph, obtained by removing the direction of every arc, is connected is said to be weakly connected. Further information on graph theory can be found in [18-20].

Throughout the rest of the paper, we assume that $X$ and $Y$ are two given topological spaces. If it is not explicitly mentioned, we consider that these spaces are endowed with the discrete topology. In this case, the unique dense set of $X$ is the whole set $X$ itself. We will also consider the product of $N$ copies of these spaces, $X^{N}$ and $Y^{N}$ with $N \in \mathbb{N}$, equipped with the usual product space topologies.

We introduce several notions of dynamical systems in the setting of binary relations:

Definition 1. Let $\left(\rho_{n}\right)_{n \in \mathbb{N}}$ be a sequence of binary relations between the spaces $X$ and $Y, \rho$ a binary relation on $X$, and $x \in X$. Then we say that

(i) $\quad x$ is a universal element for the sequence $\left(\rho_{n}\right)_{n \in \mathbb{N}}$ if $x \in \bigcap_{n \in \mathbb{N}} D\left(\rho_{n}\right)$ and for each $n \in \mathbb{N}_{0}$ there exists an element $y_{n} \in \rho_{n}(x)$ such that the set $\left\{y_{n}: n \in \mathbb{N}\right\}$ is dense in $Y$. As a particular case, if $\rho_{n}:=\rho^{n}$, then we say $x$ is hypercyclic for $\rho$.

(ii) $\rho$ is topologically transitive if, for every pair of non-empty open sets $U, V \subset X$, there is some $n \in \mathbb{N}$ such that $U \cap \rho^{-n}(V) \neq \varnothing$. If there is some $n_{0} \in \mathbb{N}$ such that this last condition holds for all $n \geq n_{0}$, we say that $\rho$ is topologically mixing.

Clearly, if $x$ is hypercyclic for $G=(X, \rho)$, and $(z, x) \in \rho^{l}$ for some $l \in \mathbb{N}$, then $z$ is likewise hypercyclic for $G$. If $\rho$ is a binary equivalence relation and $x$ is hypercyclic, then the underlying (di-)graph must be (strongly) connected and all the elements of $X$ are hypercyclic for $\rho$.

Let us consider $\left(O_{n}\right)_{n \in \mathbb{N}}$ a base of non-empty open sets for the topology of $X$. If we denote by $H C(\rho)$ the set consisting of all hypercyclic elements of $\rho$, then the following equality holds [21]:

$$
H C(\rho)=\bigcap_{n \in \mathbb{N}} \bigcup_{k \in \mathbb{N}} \rho^{-k}\left(O_{n}\right)
$$

Remark 1. (i) Let $G=(X, \rho)$ be a graph with $X$ equipped with the discrete topology. It can be simply proved that the graph $G$ is connected if and only if $\rho$ is topologically transitive or hypercyclic.

(ii) In the Definition 1 (ii), it is irrelevant whether we write $\rho^{-n}(V)$ or $\rho^{n}(V)$. It is also worth noting that $\rho$ does not need to be topologically mixing whenever $\rho$ is topologically transitive: Let us consider a graph $G=(X, \rho)$ that is isomorphic to a square, that is $X=\left\{x_{1}, x_{2}, x_{3}, x_{4}\right\}$ and $\rho=\left\{\left(x_{1}, x_{2}\right),\left(x_{2}, x_{3}\right),\left(x_{3}, x_{4}\right),\left(x_{4}, x_{1}\right)\right\}$, see Figure 1a. Clearly, $\rho$ is topologically transitive, but it does not hold the topologically mixing property since there is no odd number $n \in \mathbb{N}$ such that $x_{3} \in \rho^{n}\left(x_{1}\right)$.

(iii) Unlike the linear setting, in our framework, the notion of hypercyclicity cannot be connected to that of topological transitivity in any reasonable way. It is well known that these notions are equivalent for continuous linear operators on Fréchet spaces by Baire's category theorem (see, for instance, [21,22]). However, there exist examples of continuous linear operators on non-metrizable locally convex spaces that are topologically transitive and not hypercyclic [23]. Moreover, for any non-trivial Banach space $X$ there exists a multivalued linear operator $\mathcal{A}=\{0\} \times X$ that is hypercyclic and not topologically transitive (cf. [24]). It is very simple to construct an example of a hypercyclic relation on a finite set that is not topologically transitive, as well: Set the digraph $G=(X, \rho)$ with $X:=\left\{x_{1}, x_{2}, x_{3}\right\}$ and $\rho:=\left\{\left(x_{1}, x_{2}\right),\left(x_{2}, x_{1}\right),\left(x_{1}, x_{3}\right)\right\}$, endowing $X$ with the discrete topology, see Figure $1 b$. Then $x_{1}$ is a hypercyclic element for $\rho$, but $\rho$ is not topologically transitive, since $\left\{x_{2}\right\} \cap \rho^{n}\left(\left\{x_{3}\right\}\right)=\varnothing$ for all $n \in \mathbb{N}$. 


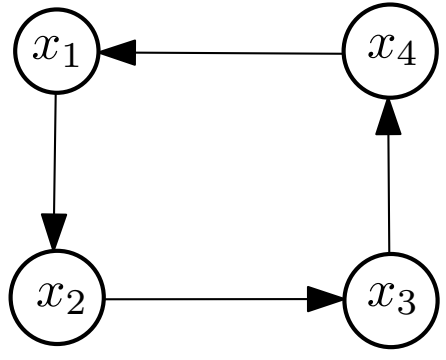

(a)

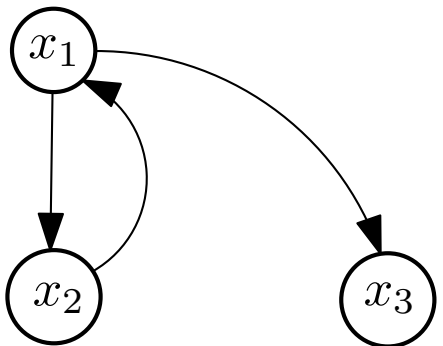

(b)
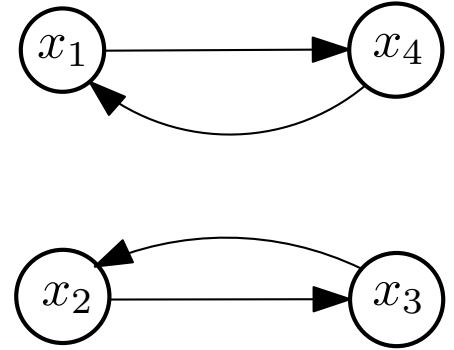

(c)

Figure 1. (a) In Remark 1 (ii), we show a graph that, endowed with the discrete topology, it is topologically transitive but not topologically mixing. (b) In Remark 1 (iii), we have a graph that, endowed with the discrete topology, it is hypercyclic but not topologically transitive. (c) In Example 1, different topologies- $\tau_{1}=\left\{\varnothing,\left\{x_{1}\right\},\left\{x_{2}\right\},\left\{x_{3}\right\},\left\{x_{1}, x_{2}\right\},\left\{x_{1}, x_{3}\right\}\right.$, $\left.\left\{x_{2}, x_{3}\right\},\left\{x_{1}, x_{2}, x_{3}\right\},\left\{x_{1}, x_{2}, x_{3}, x_{4}\right\}\right\}$ and $\tau_{2}=\left\{\varnothing,\left\{x_{1}, x_{2}\right\},\left\{x_{1}, x_{2}, x_{3}, x_{4}\right\}\right\}$-can be defined such that the graph is hypercyclic for $\tau_{1}$ but not for $\tau_{2}$.

By considering different topologies on the set of nodes $X$, the aforementioned dynamical properties can be used to generalize the notion of connectivity. The following examples illustrate these facts:

Example 1. Let $G=(X, \rho)$ be a graph without isolated nodes, and let $\tau$ be the topology on $X$. Let us denote by $G_{1}, \ldots, G_{k}$, the connected components of the graph $G$. Then $\rho$ is hypercyclic if there exists a number $i \in \mathbb{N}_{k}$ such that $G_{i}$ is dense in $(G, \tau)$, and any element of $G_{i}$ is a hypercyclic element of $\rho$.

We point out that the topology endowed to $X$ is crucial in order to ensure hypercyclicity, or any other dynamical property. For example, let us take $X=\left\{x_{1}, x_{2}, x_{3}, x_{4}\right\}$, endowed with $\tau_{1}=\left\{\varnothing,\left\{x_{1}\right\},\left\{x_{2}\right\},\left\{x_{3}\right\}\right.$, $\left.\left\{x_{1}, x_{2}\right\},\left\{x_{1}, x_{3}\right\},\left\{x_{2}, x_{3}\right\},\left\{x_{1}, x_{2}, x_{3}\right\},\left\{x_{1}, x_{2}, x_{3}, x_{4}\right\}\right\}$, and $\tau_{2}=\left\{\varnothing,\left\{x_{1}, x_{2}\right\},\left\{x_{1}, x_{2}, x_{3}, x_{4}\right\}\right\}$, see Figure 1c. We now set the binary relation $\rho:=\left\{\left(x_{1}, x_{4}\right),\left(x_{4}, x_{1}\right),\left(x_{2}, x_{3}\right),\left(x_{3}, x_{2}\right)\right\}$, then $\rho$ is not hypercyclic in $\left(X, \tau_{1}\right)$ but it is hypercyclic in $\left(X, \tau_{2}\right)$.

Finally, observe that $\rho$ is topologically transitive (and $\rho$ is topologically mixing) if, for every pair of non-empty open subsets of $U, V \subset X$, there exists $i \in \mathbb{N}_{k}$ such that $U \cap G_{i} \neq \varnothing$ and $V \cap G_{i} \neq \varnothing$.

The links between connectivity and dynamics will be thoroughly explained in the next section.

\section{Hypercyclic and Chaotic Digraphs}

We study the relations between different types of connectivity on digraphs and some of the aforementioned dynamical properties. This will enable us to introduce two new important classes of digraphs that are subclasses of the class of weakly connected digraphs and that extend the class of the strongly connected ones.

One of the most accepted notions of chaos is the one introduced by Devaney [25] for continuous mappings acting on metric spaces. Three ingredients are considered in this definition: topological transitivity, density of periodic points, and sensitive dependence on the initial conditions (SDIC). Banks et al. [26] proved that SDIC can be deduced from the other two properties. Nevertheless, since topological transitivity does not coincide with hypercyclicity in our setting, we will introduce two different notions of chaos.

Definition 2. Let $\rho$ be a binary relation on $X$, and $x \in X$. Then we say that

1. $x$ is a periodic point of $\rho$ if $x \in D_{\infty}(\rho)$ and there exists $n \in \mathbb{N}$ such that $x \in \rho^{n}(x)$.

2. $\rho$ is Devaney-chaotic if it is topologically transitive and it has a dense set of periodic points.

3. $\rho$ is chaotic if it is hypercyclic and it has a dense set of periodic points. 
Let us consider $G=(X, \rho)$, where $X$ is endowed with the discrete topology. It immediately follows from our definitions that $G$ is strongly connected if and only if $\rho$ is topologically transitive. Every $x \in X$ is also a periodic element for $\rho$. Thus, $\rho$ is also Devaney-chaotic. Besides, $\rho$ is also hypercyclic and chaotic, and $x \in H C(\rho)$ for every $x \in X$. Since $\rho$ is hypercyclic, let us pick any $x \in H C(\rho)$. Then, for any two different points $y, z \in X$, an $x-y$ path and an $x-z$ path exists in $G$. Thus, considering the underlying non-directed edges, there exists a walk connecting $y$ and $z$, and $G$ is thus weakly connected. We summarize all these relations underneath.

$(G, \rho)$ is Devaney-chaotic (equiv., strongly connected) $\Rightarrow(G, \rho)$ is chaotic $\Rightarrow(G, \rho)$ is hypercyclic $\Rightarrow(G, \rho)$ is weakly connected.

Therefore, we are able to introduce two new classes of digraphs that are subclasses of the class of weakly connected digraphs and that extend the notion of strong connectivity. Any of these three implications is strict, as the following examples show:

Example 2. (i) Let $X:=\left\{x_{1}, x_{2}, x_{3}, x_{4}\right\}$ be equipped with discrete topology, and let $\rho:=$ $\left\{\left(x_{1}, x_{2}\right),\left(x_{2}, x_{1}\right),\left(x_{1}, x_{3}\right),\left(x_{3}, x_{4}\right),\left(x_{4}, x_{3}\right)\right\}$, see Figure $2 a$. Then $x_{3} \in \rho\left(x_{1}\right), x_{4} \in \rho^{2}\left(x_{1}\right), x_{1} \in$ $\rho^{3}\left(x_{1}\right), x_{2} \in \rho^{2 n+1}\left(x_{1}\right)$, and $x_{1} \in \rho^{2 n}\left(x_{1}\right)(n \geq 2)$, which simply yields that $x_{1} \in H C(\rho)$. It is also clear that any element of $X$ is periodic for $\rho$, such that $\rho$ is chaotic. Since there is no path connecting $x_{3}$ and $x_{1}$ in $G, G$ is not strongly connected and $\rho$ is neither topologically transitive nor Devaney-chaotic. Therefore, the first implication in Equation (2) is strict.

(ii) Let $C=x_{1} \ldots x_{n+1}$ be an oriented closed cycle of length $n$ (with $x_{1}=x_{n+1}$ ), and let $\rho:=C \cup$ $\left\{\left(x_{1}, x_{n+2}\right)\right\}$, where $x_{n+2} \neq x_{j}$ for $1 \leq j \leq n+1$, see Figure $2 b$. Then it can be easily seen that $G=(X, \rho)$, endowed with the discrete topology, is hypercyclic, since any element lying on the cycle $C$ is hypercyclic for $\rho$ and that $G$ is not chaotic, because the point $x_{n+2}$ cannot be a periodic element for $\rho$.

(iii) Let $X:=\left\{x_{1}, x_{2}, x_{3}\right\}$ and $\rho:=\left\{\left(x_{1}, x_{2}\right),\left(x_{3}, x_{2}\right)\right\}$, see Figure $2 c$. Then $G=(X, \rho)$ is weakly connected but, equipped with the discrete topology, it is not hypercyclic.

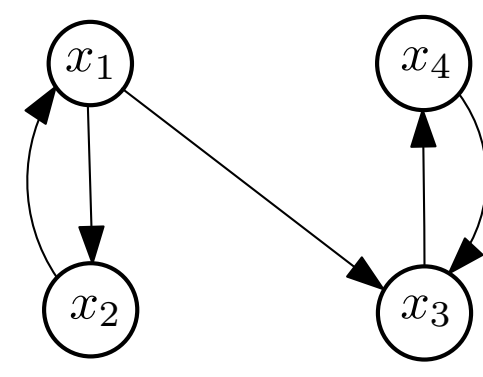

(a)

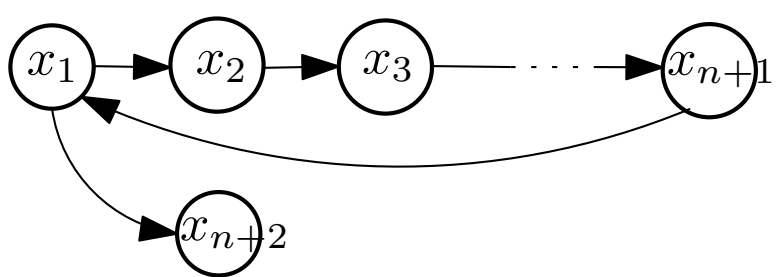

(b)

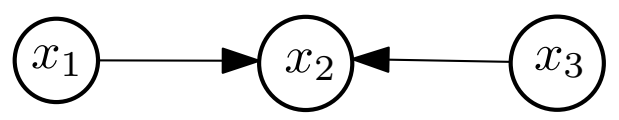

(c)

Figure 2. The following graphs are endowing with the discrete topology. (a) In Example 2 (i), we show that all in the elements in a graph can be periodic, but this does not imply strong connectivity, Devaney chaos, nor topological transitivity. (b) In Example 2 (ii), we show that hypercyclicity does not imply density of periodic points. (c) In Example 2 (iii), we show that weak connectivity does not imply hypercyclicity.

A series of recent results in the theory of digraphs are devoted to the study of cyclability: Given a digraph $G=(X, \rho)$, a set $S \subset X$ said to be cyclable in $G$ if $G$ contains a cycle through all the nodes of $S$. 
Setting $S=X$, we obtain the classical concept of a Hamiltonian (di-)graph. We refer the interested reader to [27]. It is clear that the cyclability of $S \subset X$ in $G=(X, \rho)$ jointly with the denseness of $S$ in $X$ imply that $\rho$ is (Devaney)-chaotic for $\rho$. However, this is far from being necessary for $\rho$ to be (Devaney)-chaotic since this condition is automatically satisfied if the points in $S$ lie on a closed path in $G$ not on a circle.

It is worth noting that our conclusions from Example 1 can be reformulated for digraphs only partially; if $G_{1}, G_{2}, \ldots, G_{k}$ denote the strongly connected components of a digraph $G=(X, \rho)$, then the denseness of some $G_{i}$ in $G$ implies that $\rho$ is (Devaney)-chaotic for $\rho$, but the converse statement fails to be true even for discrete topology, as the next example shows:

Example 3. Consider a digraph $G$ consisting of two oriented cycles given by $G_{1}:=\left\{x_{1}, x_{2}, x_{3}\right\}$ and $G_{2}:=$ $\left\{x_{4}, x_{5}, x_{6}\right\}$ joined by an arc $\left(x_{1}, x_{4}\right)$, see Figure $3 a$. Then $G$ is Devaney-chaotic but neither $G_{1}$ nor $G_{2}$ are dense in $G$.

\section{Dynamics on Tournaments}

Without any doubt, tournaments are the best studied class of digraphs (see, for instance, the classical reference of [28]). We recall their definition.

Definition 3. A tournament $T=(X, \rho)$ is a digraph in which any pair of different nodes $x, y \in X$ are connected by exactly one arc.

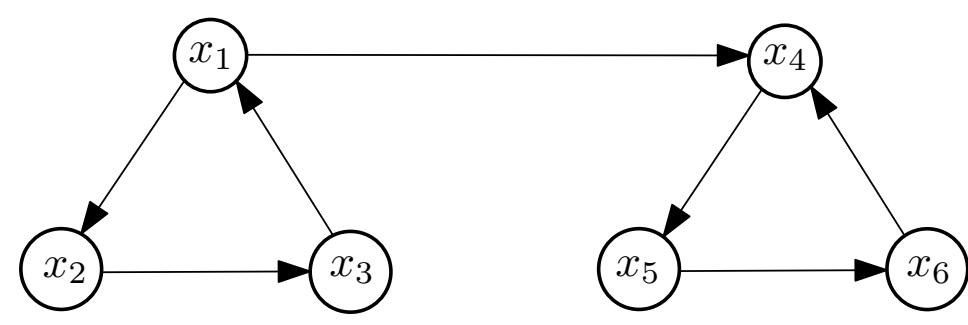

(a)

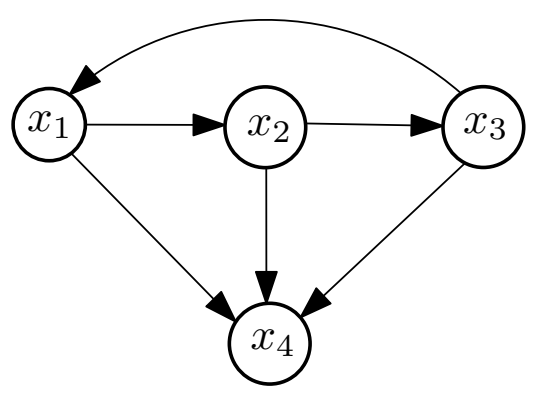

(b)

Figure 3. (a) In Example 3, we show a Devaney-chaotic digraph, whose strongly connected components are not dense. (b) In Example 4, we show an example of a tournament that, endowed with the discrete topology, it is hypercyclic but not topologically transitive.

Example 4. It is straightforward that a tournament $T=(X, \rho)$ with $X=\left\{x_{1}, x_{2}, x_{3}, x_{4}\right\}$ and $\rho$ obtained as the union of a cycle $\left\{\left(x_{1}, x_{2}\right),\left(x_{2}, x_{3}\right),\left(x_{3}, x_{1}\right)\right\}$ with the $\left.\operatorname{arcs}\left\{\left(x_{1}, x_{4}\right),\left(x_{2}, x_{4}\right),\left(x_{3}, x_{4}\right)\right\}\right)$ is hypercyclic but not topologically transitive, see Figure $3 b$.

In the following theorem, we completely characterize the class of hypercyclic tournaments.

Theorem 1. Let $T_{n}=(X, \rho)$, with $|X|=n$, be a tournament of $n$ nodes equipped with the discrete topology. Then $T_{n}$ is hypercyclic if and only if $d^{+}(x)<n-1$ for every $x \in X$.

Proof. First, assume that $T_{n}$ is hypercyclic and $d^{+}(x)=n-1$ for some $x \in X$. Then $d^{-}(x)=0$, and there is no $k \in \mathbb{N}, z \in\left\{x_{1}, x_{2}, \ldots, x_{n}\right\}$ such that $x \in \rho^{k}(z)$, which contradicts the hypercyclicity of $T_{n}$. Now, let us assume that the outdegree of any vertex is strictly less than $n-1$. As a consequence of the famous theorem attributed to Rédei [29], there exists a non-closed path visiting all nodes of $X$, that is a Hamiltonian path named $x_{1}, x_{2} \ldots, x_{n}$. Therefore, $x_{1} \in H C(\rho)$. 
It is clear that, in any arbitrary tournament $T$, we have that $d^{+}(x)+d^{-}(x)=n-1$ for all $x \in X$, and $d^{+}(x)<n-1$ if and only if $d^{-}(x)>0$. For digraphs, we can prove the following extension of Theorem 1:

Corollary 1. Let $G=(X, \rho)$ be a digraph and $X$ equipped with discrete topology, with $|X|=n \geq 3$. Suppose that, for any pair of nodes, $x, y \in X$ such that, if $(x, y) \notin \rho$, we have $d^{+}(x)+d^{-}(y) \geq n-1$. Then $\rho$ is hypercyclic if and only if $d^{-}(x)>0$ for all $x \in X$.

Proof. It is clear that the hypercyclicity of $\rho$ implies that the indegree of any node is strictly positive. The converse statement follows from the fact that such assumptions imply that there is a Hamiltonian path in $G$ by Ore's Theorem [20], and the argumentation is in the proof of Theorem 1.

\section{Disjointness on Binary Relations, Graphs, and Digraphs}

Disjointness was firstly introduced for dynamical systems by Furstenberg in [30]. For linear operators, it was firstly considered in [31,32] (see also [33]). Further information on the dynamics of linear operators can be found in [34-37]. We introduce analogons of disjointness of the above classes of hypercyclic and (Devaney)-chaotic graphs. Part of these results are inspired by the analogons obtained for the case of multivalued linear operators in [5].

We first introduce the notion of disjointness for hypercyclicity, topological transitivity, and the topologically mixing property.

Definition 4. Let $N \geq 2$. Let $X, Y$ be two topological spaces. For every $j \in \mathbb{N}_{N}$, let $\left(\rho_{j, n}\right)_{n \in \mathbb{N}}$ be a sequence of binary relations between the spaces $X$ and $Y$, let $\rho_{j}$ be a binary relation on $X$, and let $x \in X$. An element $x \in X$ is a d-universal element for the sequences $\left(\rho_{1, n}\right)_{n \in \mathbb{N}}, j \in \mathbb{N}_{N}$ if, for each $j \in \mathbb{N}_{N}, n \in \mathbb{N}$, there exist elements $y_{j, n} \in \rho_{j, n}(x)$ such that the set $\left\{\left(y_{1, n}, y_{2, n}, \ldots, y_{N, n}\right): n \in \mathbb{N}\right\}$ is dense in $Y^{N}$. As a particular case, the binary relations $\rho_{1}, \ldots, \rho_{N}$ are called $\mathrm{d}$-hypercyclic if there exists a $d$-universal element $x$ of the sequences $\left(\rho_{1}^{n}\right)_{n \in \mathbb{N}}, \ldots,\left(\rho_{N}^{n}\right)_{n \in \mathbb{N}}$. In this case, $x$ is called a d-hypercyclic element of the binary relations $\rho_{1}, \ldots, \rho_{N}$.

Definition 5. Let $N \geq 2$. Let $X, Y$ be two topological spaces. For every $1 \leq j \leq N$, let $\left(\rho_{j, n}\right)_{n \in \mathbb{N}}$ be a sequence of binary relations between the spaces $X$ and $Y$ and let $\rho_{j}$ be a binary relation on $X$.

1. The sequences $\left(\rho_{1, n}\right)_{n \in \mathbb{N}}, \ldots,\left(\rho_{N, n}\right)_{n \in \mathbb{N}}$ are $\mathrm{d}$-topologically transitive if, for every non-empty open subsets $U \subset X$ and $V_{1}, \ldots, V_{N} \subset Y$, there exists $n \in \mathbb{N}$ such that

$$
U \cap \rho_{1, n}^{-1}\left(V_{1}\right) \cap \ldots \cap \rho_{N, n}^{-1}\left(V_{N}\right) \neq \varnothing .
$$

2. The sequences $\left(\rho_{1, n}\right)_{n \in \mathbb{N}}, \ldots,\left(\rho_{N, n}\right)_{n \in \mathbb{N}}$ are $\mathrm{d}$-topologically mixing if, for every non-empty open subsets $U \subset X$ and $V_{1}, \ldots, V_{N} \subset Y$, there exists $n_{0} \in \mathbb{N}$ such that, for every $n \geq n_{0}$, we have that Equation (3) holds.

3. The binary relations $\rho_{1}, \cdots, \rho_{N}$ are $d$-topologically transitive (d-topologically mixing) if the sequences $\left(\rho_{1}^{n}\right)_{n \in \mathbb{N}}, \ldots,\left(\rho_{N}^{n}\right)_{n \in \mathbb{N}}$ are d-topologically transitive (d-topologically mixing).

We also introduce the notion of d-Devaney chaos and d-chaos for binary relations. For this purpose, we define the set of periodic elements

$$
\mathcal{P}\left(\rho_{1}, \rho_{2}, \cdots, \rho_{N}\right):=\left\{\left(x_{1}, x_{2}, \ldots, x_{N}\right) \in X^{N}: \exists n \in \mathbb{N} \text { with } x_{j} \in \rho_{j}^{n}\left(x_{j}\right), j \in \mathbb{N}_{N}\right\} .
$$

Definition 6. Given $N \geq 2$, the binary relations $\rho_{1}, \ldots, \rho_{N}$ on $X$ are said to be $\mathrm{d}$-Devaney-chaotic if they are $d$-topologically transitive and the set of periodic elements $\mathcal{P}\left(\rho_{1}, \rho_{2}, \cdots, \rho_{N}\right)$ is dense in $X^{N}$. These relations are d-chaotic if they are d-hypercyclic and the set of periodic elements is dense in $X^{N}$. 
A formula similar to Equation (1) can be given for d-hypercyclic elements of binary relations [32], and the most important consequences of both formulae can be formulated for continuous mappings acting between topological spaces.

It is well known that two single-valued linear operators acting on a Fréchet space cannot be $\mathrm{d}$-hypercyclic if one of them is a scalar multiple of the other one. This is no longer true for multivalued linear operators since there exists a multivalued linear operator $\mathcal{A}$ on a Banach space such that $\mathcal{A}$ and some arbitrary multiples of it can be d-hypercyclic [5]. Concerning simple graphs, the notion of d-hypercyclicity is much more complicated than the notion of hypercyclicity, and it does not reduce to the connectivity of the graphs, as is described in Example 2 (ii). For the next result, we recall that a graph of $n$ nodes $K_{n}=\left(X_{n}, \rho\right)$ is complete if, for every pair of different nodes $x, y \in X_{n}$, we have that $(x, y) \in \rho$, i.e., $\rho=X \times X \backslash \Delta_{X}$.

Theorem 2. Let $n \geq 2$, and let $K_{n}=\left(X_{n}, \rho\right)$ denote the complete graph with $X_{n}=\left\{x_{1}, x_{2}, \ldots, x_{n}\right\}$, equipped with discrete topology. Let us consider $N \geq 2$ copies of the graphs $K_{n}$. The following affirmations are equivalent:

\section{i. The graphs are d-Devaney-chaotic. \\ ii. The graphs are d-hypercyclic. \\ iii. Each graph contains $n \geq 3$ nodes.}

Proof. Clearly, (i) implies (ii). To prove (ii) implies (iii), we only need to observe that, in the case $n=2$, we do not have the existence of a natural number $k \in \mathbb{N}$ such that $x_{1} \in \rho^{k}\left(x_{1}\right)$ and $x_{2} \in \rho^{k}\left(x_{1}\right)$. Thus, any $N$-tuple of the $X_{2}^{N}$ containing two different components cannot be an element of the set $\cup_{k \in \mathbb{N}}\left[\left(\rho^{k}\left(x_{1}\right)\right)^{N} \cup\left(\rho^{k}\left(x_{2}\right)\right)^{N}\right]$. Hence, neither $x_{1}$ nor $x_{2}$ are d-hypercyclic elements for the $N$ graphs $K_{2}$. Now, let us assume $n \geq 3$ and let us see that the $N$ copies of $K_{n}$ are d-Devaney-chaotic. For any $N$-tuple $\left(x_{i_{1}}, \ldots, x_{i_{N}}\right) \in X_{n}^{N}$ and $i \in \mathbb{N}_{N}$, we have that $\left(x_{i_{1}}, \ldots, x_{i_{N}}\right) \in\left(\rho^{2}\left(x_{i}\right)\right)^{N}$ so that $x_{i}$ is a d-hypercyclic element for the $N$-tuple of graphs $K_{n}$. Finally, the set of periodic points $\mathcal{P}(\rho, \ldots, \rho)$ coincides with $X_{n}^{N}$, even in the case $n=2$.

In a similar way, it can be seen the equivalence of d-chaos and d-transitivity with the condition $n \geq 3$.

Another remarkable class of graphs are bipartite graphs. A graph $G=(X, \rho)$ is said to be bipartite if $X=X_{1} \cup X_{2}$ with $X_{1} \cap X_{2}=\varnothing$ and for every $(x, y) \in \rho$, either $x \in X_{1}$ and $y \in X_{2}$ or $x \in X_{2}$ and $y \in X_{1}$. In this case, the d-hypercyclicity can never hold. If we consider a family of bipartite graphs $G_{1}, \ldots, G_{N}$, sharing the partition of the set of nodes $X$ into two sets $X_{1}$ and $X_{2}$, then for any $x \in X$ and every $k \in \mathbb{N}$ either $\rho_{1}^{k}(x) \times \ldots \times \rho_{N}^{k}(x) \subset X_{1}^{N}$ or $\rho_{1}^{k}(x) \times \ldots \times \rho_{N}^{k}(x) \subset X_{2}^{N}$, depending on where is $x$ and if $k$ is even or odd.

Before proceeding further, we would like to observe that the notion of (d-topological transitivity) $\mathrm{d}$-hypercyclicity is equivalent for simple graphs equipped with the discrete topology to the notion of d-Devaney chaos, since for any simple graph $G=(X, \rho)$ and for any $x \in X$ we have that $x \in \rho^{2 k}(x)$.

In the remaining part of this section, we will only consider digraphs.

For any digraph $G=(X, \rho)$, where $X=\left\{x_{1}, \ldots, x_{n}\right\}$, we introduce the adjacency matrix of $A$, $[A]=\left[a_{i, j}\right]$ shortly, by $a_{i, j}:=1$ if $\left(x_{i}, x_{j}\right) \in \rho$ and $a_{i, j}:=0$ if $\left(x_{i}, x_{j}\right) \notin \rho$, for every $1 \leq i, j \leq n$.

Let $G_{1}, \ldots, G_{N}$ be digraphs with, $G_{i}=\left(X_{i}, \rho_{i}\right), i \in \mathbb{N}_{N}$, each of which is equipped with discrete topology, and let $A_{1}, \ldots, A_{N}$ be their corresponding adjacency matrices. Denote, for every $l \in \mathbb{N}_{N}$ and $k \in \mathbb{N}$, the $k$-th power of the adjacency matrix $A_{l}$ as $A_{l}^{k}=\left[a_{i, j}^{l, k}\right]_{1 \leq i, j \leq n}$. As is well known, the element $a_{i, j}^{l, k}$ of matrix $A_{l}^{k}$ represents the exact number of $x_{i}-x_{j}$ walks of length $k$ in $G_{l}$. Using this result and our definition of d-hypercyclic elements, we can simply clarify the following necessary and sufficient conditions for an element to be a d-hypercyclic element.

Theorem 3. Let $G_{1} \ldots G_{N}$ be digraphs over $X=\left\{x_{1}, \ldots, x_{n}\right\}$, equipped with the discrete topology, with adjacency matrices $A_{1}, \ldots, A_{N}$. An element $x_{i} \in X$ is d-hypercyclic for $G_{1}, \ldots, G_{N}$ if and only if, for every $\left(j_{1}, j_{2}, \ldots, j_{N}\right) \in \mathbb{N}_{n}^{N}$, there exists $k \in \mathbb{N}$ such that, for all $l \in \mathbb{N}_{N}, a_{i, j_{l}}^{l, k} \geq 1$. 
By taking $V_{l}=\left\{x_{j_{l}}\right\}, l \leq \mathbb{N}_{N}$ and $U=\left\{x_{i}\right\}$ in Condition 3, we obtain the following characterization of d-topological transitivity of digraphs $G_{1}, \ldots, G_{N}$ equipped with discrete topologies, and we can also characterize d-topologically mixing property in a similar way:

Theorem 4. Let $G_{1} \ldots G_{N}$ be digraphs over $X=\left\{x_{1}, \ldots, x_{n}\right\}$, equipped with the discrete topology, with adjacency matrices $A_{1}, \ldots, A_{N}$. The digraphs $G_{1}, \ldots, G_{N}$ are d-topologically transitive if and only if, for every $\left(j_{1}, j_{2}, \ldots, j_{N}\right) \in \mathbb{N}_{n}^{N}$ and for every $i \in \mathbb{N}$, there exists $k \in \mathbb{N}$ such that, for all $l \in \mathbb{N}_{N}, a_{i, j_{l}}^{l, k} \geq 1$.

Thus, d-topological transitivity of digraphs $G_{1}, \ldots, G_{N}$ equipped with discrete topologies immediately implies that these digraphs are d-hypercyclic with any $x \in G$ being a d-hypercyclic element. It is clear that d-Devaney chaos of $G_{1}, G_{2}, \ldots, G_{N}$ implies d-chaos, which also implies d-hypercyclicity. The converse implications do not hold in general, as can be illustrated with the following examples.

Example 5. (i) Let $G$ be the graph appearing in Example 2 (i), and let $K_{4}$ be the complete graph of these 4 nodes, and both are equipped with discrete topologies, see Figure $4 a$. It can be easily seen that $G$ and $H$ are $d$-chaotic but not d-Devaney-chaotic.

(ii) Let $X:=\left\{x_{1}, x_{2}, x_{3}, x_{4}\right\}$, and let $\rho:=\bigcup_{1 \leq i, j \leq 3}\left(x_{i}, x_{j}\right) \cup\left\{\left(x_{1}, x_{4}\right),\left(x_{2}, x_{4}\right)\right\}$, see Figure $4 b$. If $G=$ $(X, \rho)$ is equipped with the discrete topology, then the pair $G, G$ is d-hypercyclic, since, for any even number $k \in \mathbb{N}$ and for every $x \in X$, we have $x \in \rho^{k}\left(x_{1}\right)$, but it is not d-chaotic since $x_{4}$ is not periodic in $G$.
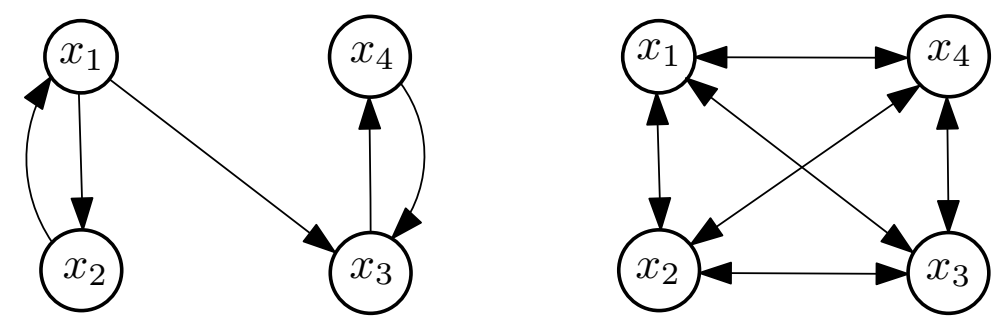

(a)
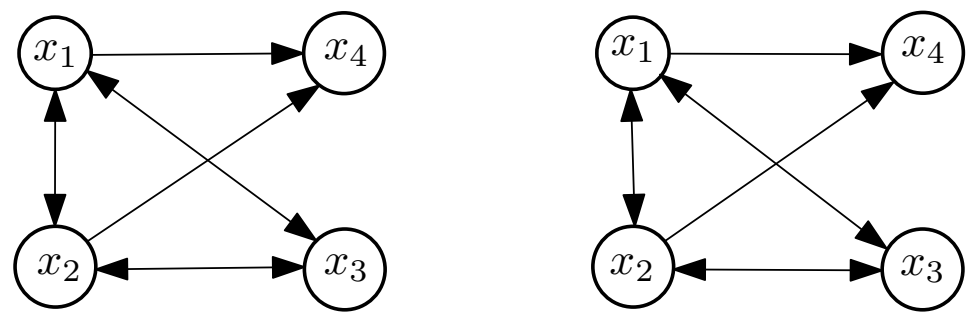

(b)

Figure 4. The following graphs are endowed with the discrete topology. (a) In Example 5 (i), we show that the graph of Example 2 (i) and $K_{4}$ are d-chaotic but not d-Devaney-chaotic. (b) In Example 5 (ii), these two copies of the same graph are d-hypercyclic but not d-chaotic.

Disjointness of tournaments depends on the size of the set of nodes.

Theorem 5. Let $n \leq 3$, and let $T_{1}, \ldots, T_{N}$ be $N$ tournaments equipped with discrete topologies. Then $T_{1}, \ldots, T_{N}$ cannot be d-hypercyclic nor d-topologically transitive.

Proof. Without loss of generality, we may assume that $N=2$. If $n=2$, then it can be easily seen that $T_{1}$ and $T_{2}$ must be isomorphic to an oriented segment, which is neither hypercyclic nor topologically transitive. 
If $n=3$, there exist only two non-isomorphic tournaments of order 3 . Since $T_{1}$ and $T_{2}$ should be hypercyclic (topologically transitive), and only the Hamiltonian circles are hypercyclic (topologically transitive), it follows that $T_{1}$ and $T_{2}$ have to be a Hamiltonian circle-either $x_{1}, x_{2}, x_{3}, x_{1}$ or $x_{1}, x_{3}, x_{2}, x_{1}$. A careful inspection of all possible cases leads us to the impossibility of satisfying any of these dynamical properties.

By Theorems 2 and 5, looking at the case $n=3$, we easily observe the existence of digraphs that are not d-chaotic, but whose underlying simple graphs are d-chaotic. In the case of $n \geq 4$, we have the following important result.

Theorem 6. Let $n \geq 4$, and let $T_{1}, \ldots, T_{N}$ be tournaments over a set $X=\left\{x_{1}, \ldots, x_{n}\right\}$ equipped with discrete topologies. The following are equivalent:

1. $T_{1}, \ldots, T_{N}$ are d-Devaney-chaotic.

2. $T_{1}, \ldots, T_{N}$ are d-topologically transitive.

3. $T_{l}$ is strongly connected for all $l \in \mathbb{N}_{N}$.

4. $T_{l}$ is a Hamiltonian tournament for all $l \in \mathbb{N}_{N}$.

Proof. Let $T_{1}, \ldots, T_{N}$ be d-chaotic. Then these tournaments are d-topologically transitive such that any $T_{l}, l \in \mathbb{N}_{N}$, is topologically transitive and therefore strongly connected. By Camion's theorem (see, for instance, [28]), $T_{l}$ is strongly connected if and only if each $T_{l}$ is Hamiltonian.

Therefore, it remains to be proven that the strong connectivity of all $T_{l}$ values implies that $T_{1}, \ldots, T_{N}$ are d-chaotic. To see this, let us recall that the strong connectivity of any directed graph is equivalent to its irreducibility [38]. Applying Th. 1 of [38], we have that the adjacency matrix $A_{l}$ of $T_{l}, l \in \mathbb{N}_{N}$, is primitive, i.e., there exists a natural number $q_{l}$ such that $A_{l}^{q_{l}}$ is strictly positive. In fact, Wielandt estimates that $q_{l} \leq(n-1)^{2}+1$ [39]. The result now follows from an application of Theorem 4 and from the observation that, for a given tuple $\left(j_{1}, j_{2}, \ldots, j_{N}\right) \in \mathbb{N}_{n}^{N},\left(x_{j_{1}}, x_{j_{2}}, \ldots, x_{j_{N}}\right)$ is a periodic point for $T_{1}, \ldots, T_{N}$ if and only if there exists $k \in \mathbb{N}$ such that, for each $l \in \mathbb{N}_{N}$, one has $a_{j_{l}, j_{l}}^{l, k} \geq 1$.

Any of the above equivalencies implies that $T_{1}, \ldots, T_{N}$ are also d-hypercyclic. However, the situation is not so simple because the strong connectivity of $T_{l}$ values for all $l \in \mathbb{N}_{N}$ is not equivalent to the fact that $T_{1}, \ldots, T_{N}$ were d-hypercyclic:

Example 6. It is well known that there exist only four non-isomorphic tournaments of order four. Only two of them, $T_{1}$ and $T_{2}$, defined as follows, are hypercyclic: $T_{1}$ is the union of the Hamiltonian cycle $x_{2}, x_{3}, x_{4}, x_{2}$ and oriented segments $\left(x_{2}, x_{1}\right),\left(x_{3}, x_{1}\right)$, and $\left(x_{4}, x_{1}\right)$, while $T_{2}$ is the union of the Hamiltonian cycle $x_{1}, x_{2}, x_{3}, x_{4}, x_{1}$ and oriented segments $\left(x_{1}, x_{3}\right)$ and $\left(x_{2}, x_{4}\right)$. Furthermore, $T_{1}$ is hypercyclic but not topologically transitive, whereas $T_{2}$ is. It can be verified that the pair $T_{1}, T_{1}$ is not d-hypercyclic. Hence, the hypercyclicity of components does not imply d-hypercyclicity of a tuple. We also point out that the pair $T_{1}, T_{2}$ is d-hypercyclic, but $T_{1}$ is not strongly connected.

We close this work by providing one more example.

Example 7. Let $n \geq 5$, and $T_{1}, \ldots T_{N}$ be tournaments over a set of nodes $X=\left\{x_{1}, \ldots, x_{n}\right\}$. Suppose that $T_{1}$ is a hypercyclic, non-Hamiltonian tournament and that, for $l=2, \ldots, N, T_{l}$ is a Hamiltonian tournament. Let $x$ be a hypercyclic element for $T_{1}$. Then the proof of Theorem 6 shows that $T_{1}, \ldots T_{N}$ are d-hypercyclic, where $x$ is their d-hypercyclic element. However, it is clear that $T_{1}, \ldots T_{N}$ cannot be d-topologically transitive.

Author Contributions: All the authors have equally participated in the work.

Acknowledgments: The second and fourth authors were supported by MEC Project MTM2016-75963-P. 
Conflicts of Interest: The authors declare no conflict of interest. The founding sponsors had no role in the design of the study; in the collection, analyses, or interpretation of data; in the writing of the manuscript, and in the decision to publish the results.

\section{References}

1. Strogatz, S.H. Nonlinear Dynamics and Chaos: With Applications to Physics, Biology, Chemistry, and Engineering (Studies in Nonlinearity). In Nonlinear Dynamics and Chaos: With Applications to Physics, Biology, Chemistry, and Engineering (Studies in Nonlinearity), 2nd ed.; Addison-Wesley Publishing Company: Boston, MA, USA, 1994.

2. Hilborn, R.C. Chaos and Nonlinear Dynamics: An Introduction for Scientists and Engineers; Oxford University Press: Oxford, UK, 2000.

3. Perc, M. Visualizing the attraction of strange attractors. Eur. J. Phys. 2005, 26, 579. [CrossRef]

4. Kodba, S.; Perc, M.; Marhl, M. Detecting chaos from a time series. Eur. J. Phys. 2004, 26, 205. [CrossRef]

5. Chen, C.-C.; Conejero, J.A.; Kostić, M.; Murillo-Arcila, M. Dynamics of multivalued linear operators. Open Math. 2017, 15, 948-958. [CrossRef]

6. Bahi, J.M.; Couchot, J.-F.; Guyeux, C.; Richard, A. On the link between strongly connected iteration graphs and chaotic Boolean discrete-time dynamical systems. In Fundamentals of Computation Theory; Lecture Notes in Computer Science; Springer: Heidelberg, Germany, 2011; Volume 6914, pp. 126-137.

7. Contassot-Vivier, S.; Couchot, J.-F.; Guyeux, C.; Heam, P.-C. Random walk in a N-cube without Hamiltonian cycle to chaotic pseudorandom number generation: Theoretical and practical considerations. Int. J. Bifurc. Chaos 2017, 27, 1750014. [CrossRef]

8. Bakiri, M.; Couchot, J.-F.; Guyeux, C. One random jump and one permutation: Sufficient conditions to chaotic, statistically faultless, and large throughput prng for fpga. arXiv 2017, arXiv:1706.08093.

9. Bahi, J.M.; Guyeux, C. Discrete Dynamical Systems and Chaotic Machines: Theory and Applications; CRC Press: Boca Raton, FL, USA, 2013.

10. Guyeux, C.; Wang, Q.; Fang, X.; Bahi, J. Introducing the truly chaotic finite state machines and their applications in security field. arXiv 2017, arXiv:1708.04963.

11. Chen, C.-C. Hypercyclic and chaotic operators on $l^{p}$ spaces of Cayley graphs. Preprint, 2017.

12. Martínez-Avendano, R.A. Hypercyclicity of shifts on weighted $L^{p}$ spaces of directed trees. J. Math. Anal. Appl. 2017, 446, 823-842. [CrossRef]

13. Marijuán, C. Finite topologies and digraphs. Proyecciones 2010, 29, 291-307. [CrossRef]

14. Banasiak, J. Chaos in Kolmogorov systems with proliferation-General criteria and applications. J. Math. Anal. Appl. 2011, 378, 89-97. [CrossRef]

15. Banasiak, J.; Moszyński, M. Dynamics of birth-and-death processes with proliferation—Stability and chaos. Discret. Contin. Dyn. Syst. 2011, 29, 67-79.

16. Aroza, J.; Peris, A. Chaotic behaviour of birth-and-death models with proliferation. J. Differ. Equ. Appl. 2012, 18, 647-655. [CrossRef]

17. Namayanja, P. Chaotic phenomena in a transport equation on a network. Discret. Contin. Dyn. Syst. Ser. B 2018, in press.

18. Bondy, J.A.; Murty, U.S.R. Graph Theory with Applications; American Elsevier Publishing Co., Inc.: New York, NY, USA, 1976.

19. Chartrand, G.; Lesniak, L.; Zhang, P. Graphs E Digraphs, 6th ed.; Textbooks in Mathematics; CRC Press: Boca Raton, FL, USA, 2016.

20. Petrović, V. Graph Theory; University of Novi Sad: Novi Sad, Serbia, 1998.

21. Grosse-Erdmann, K.-G. Universal families and hypercyclic operators. Bull. Am. Math. Soc. (N.S.) 1999, 36, 345-381. [CrossRef]

22. Godefroy, G.; Shapiro, J.H. Operators with dense, invariant, cyclic vector manifolds. J. Funct. Anal. 1991, 98, 229-269. [CrossRef]

23. Bonet, J.; Frerick, L.; Peris, A.; Wengenroth, J. Transitive and hypercyclic operators on locally convex spaces. Bull. Lond. Math. Soc. 2005, 37, 254-264. [CrossRef]

24. Cross, R. Multivalued Linear Operators; Monographs and Textbooks in Pure and Applied Mathematics; Marcel Dekker, Inc.: New York, NY, USA, 1998; Volume 213. 
25. Devaney, R.L. An Introduction to Chaotic Dynamical Systems, 2nd ed.; Addison-Wesley Studies in Nonlinearity; Addison-Wesley Publishing Company, Advanced Book Program: Redwood City, CA, USA, 1989.

26. Banks, J.; Brooks, J.; Cairns, G.; Davis, G.; Stacey, P. On Devaney's definition of chaos. Am. Math. Mon. 1992, 99, 332-334. [CrossRef]

27. Bermond, J.-C.; Thomassen, C. Cycles in digraphs-A survey. J. Graph Theory 1981, 5, 1-43. [CrossRef]

28. Moon, J.W. Topics on Tournaments; Holt, Rinehart and Winston: New York, NY, USA; Montreal, QC, Canada; London, UK, 1968.

29. Gross, J.L.; Yellen, J. Graph Theory and Its Applications, 2nd ed.; Discrete Mathematics and Its Applications (Boca Raton); Chapman \& Hall/CRC: Boca Raton, FL, USA, 2006.

30. Furstenberg, H. Disjointness in ergodic theory, minimal sets, and a problem in Diophantine approximation. Math. Syst. Theory 1967, 1, 1-49. [CrossRef]

31. Bernal-González, L. Disjoint hypercyclic operators. Stud. Math. 2007, 182, 113-131. [CrossRef]

32. Bès, J.; Peris, A. Disjointness in hypercyclicity. J. Math. Anal. Appl. 2007, 336, 297-315. [CrossRef]

33. Bès, J.; Martin, Ö.; Peris, A.; hkarin, S. Disjoint mixing operators. J. Funct. Anal. 2012, 263, $1283-1322$. [CrossRef]

34. Bayart, F.; Matheron, É. Dynamics of Linear Operators; Cambridge Tracts in Mathematics; Cambridge University Press: Cambridge, UK, 2009; Volume 179.

35. Grosse-Erdmann, K.-G.; Peris, A. Linear Chaos; Universitext; Springer: London, UK, 2011.

36. Bernal-González, L.; Pellegrino, D.; Seoane-Sepúlveda, J.B. Linear subsets of nonlinear sets in topological vector spaces. Bull. Am. Math. Soc. (N.S.) 2014, 51, 71-130. [CrossRef]

37. Aron, R.M.; Bernal-González, L.; Pellegrino, D.; Seoane-Sepúlveda, J.B. Lineability: The Search for Linearity in Mathematics, Monographs and Research Notes in Mathematics; CRC Press: Boca Raton, FL, USA, 2016.

38. Moon, J.W.; Pullman, N.J. On the powers of tournament matrices. J. Comb. Theory 1967, 3, 1-9. [CrossRef]

39. Wielandt, H. Unzerlegbare, nichtnegative Matrizen. Math. Z. 1950, 52, 642-648. [CrossRef]

(C) 2018 by the authors. Licensee MDPI, Basel, Switzerland. This article is an open access article distributed under the terms and conditions of the Creative Commons Attribution (CC BY) license (http:/ / creativecommons.org/licenses/by/4.0/). 